\title{
Systematic approach in hybridization of an hydraulic skid loader
}

\author{
A. Bertini' ${ }^{2}$, M. Ceraolo ${ }^{1}$, G. Lutzemberger ${ }^{*}$
}

${ }^{1}$ Department of Energy, Systems, Territory and Constructions Engineering, University of Pisa, Largo Lucio Lazzarino n. 1, 56123, Pisa, Italy

${ }^{2}$ IMER Group, Via Salceto n. 53-55, 53036, Poggibonsi (Siena), Italy

*Corresponding author: tel. +39050 2217384, email: lutzemberger@dsea.unipi.it

\begin{abstract}
In response to growing concerns on the environment and the need to save energy, working machine manufactures have started to consider hybridization of their products. This paper presents a systematic modelling approach to identify the most promising hybrid configurations of existing skid loaders. This approach involves accurate modelling of the existing version (fitted with a conventional power train), model validation by matching the simulation results with experimental tests, design by modelling of different hybrid configurations and corresponding cost/benefit analysis.
\end{abstract}

Keywords: Skid-loader; Working machine; Hydraulics; Powertrain modelling; Hybrid propulsion system; Lithium battery.

\section{List of acronyms}

AUX: Auxiliary loads

BSFC: Brake Specific Fuel Consumption

ED: Electric Drive

EG: Electricity Generator

EM: Electric machine

EPC: Electronic Power Conditioner

ICE: Internal Combustion Engine

PC: Primary Converter 
PMM: Power Management Module

RESS: Rechargeable Energy Storage System

SOC: State of Charge

\section{Introduction}

In response to growing concerns for environmental problems and enhanced need to save energy, the demand for more environmentally friendly and fuel efficient working machines in recent years has rapidly increased [1]. In particular, during typical working operations, conventional skid loaders frequently stop and start, which generate significant amounts of braking energy that is normally converted into heat and thus lost in frictional or hydraulic braking systems [2]. Several energy saving options can be considered to recover, store and reuse this energy. A lot of research has been carried out in this direction, as detailed in [3], [4], [5], that has confirmed the validity of the usage of hybrid power trains also in the field of construction machinery.

This paper reports on the design and the development of different electric hybrid versions of an existing skid loader. The models were created using two different software packages, LMS Imagine.Lab AMESim [6] and 3DS Dymola [7]: both allow object-oriented modelling. The first one allows a detailed and fully customizable component modelling using standard program languages, or even import and use MatlabSimulink files; the other one is based on Modelica language [8], which allows the users to create their own models and comes with a great deal of totally open, well tested models and libraries from all the fields of engineering (electric, magnetic, mechanic, thermal hydraulics, etc.).

To inspire readers with confidence in the quality of the models, firstly the existing skid loader was modelled and validated by matching the simulation results and experimental tests. Later, new models were developed for different hybrid configurations, i.e. configurations in which electric power is combined with conventional (either hydraulic or mechanic or both) powers. The latter have been simulated on realistic duty cycles, defined in collaboration with the manufacturer. 


\section{The conventional skid-loader}

The conventional AS12 skid loader manufactured by Ihimer is shown in Figure 1. Its main characteristics are summarized in Table 1. It is a small working machine normally employed to transport materials (i.e. soil or rubble) within construction sites. The conventional powertrain is composed by an ICE flanged to two independent pumps that, in turn, feed through hydraulic circuits two independent hydraulic motors driving the left and right part of the vehicle. In fact, the skid loader is steered by differential speed of the two hydrostatic transmissions, and usage of independent pumps for each side is required. Another hydraulic circuit, in which a third pump is used, is aimed to feed the two linear actuators. A schematic representation of the hydraulic scheme of the skid loader is pictorially represented in Figure 2. It shows that the ICE is flanged to three pumps. The two main pumps have variable displacement and are bidirectional, while the two hydraulic motors have fixed displacement. The other fixed gears pump supplies the linear hydraulic actuators, that in pairs move the kinematic mechanism, composed by the arm and the bucket. It must however be specified that the scheme of Figure 2, in comparison to the real scheme, has been simplified giving relevance to the main components, and neglecting some other parts, i.e. details of the boost and of the oil conditioning section.

The hydraulic circuit used for propulsion contains some check and relief valves. The check valves are either wide open or completely shut, having a minimum or maximum hydraulic resistance respectively. The other type of valve are modulating valves: they usually contain a closed loop analogue control system to control the flowing fluid flow or pressure and to vary their resistance according to the flow rate or pressure differential [9]. These valves are normally used in pairs to maintain the pressure within a desired band, typically between 200 bar, i.e. the maximum allowed value for the hydraulic circuit under indication of the manufacturer for the considered machine, and 20 bar. The gear pump is used to supply both working hydraulics and boost section for the two hydrostatic transmissions, in order to reduce costs and number of components.

On the other side, the hydraulic circuit feeding the actuators is based on the action of spool valves: the basic part is constituted by one or more metering orifices with variable cross-section areas able, through the spool action, to change the direction of the flow towards one or the other of the actuator chambers. 
It must be specified that these last are proportional control valves, and they are able to perform pressure-flow rate metering according to the system architecture.

\subsection{Modelling subsystems}

The proposed models of the skid loader were built on both AMESim and Dymola. The results of simulation were comparable, which give the authors confidence in the quality of the models. All subsystems have been modelled weighting accuracy and complexity for the purpose considered. Examples of hydrostatic simulation models can be found also in [10], [11], [12], [13] also in reference to the powertrain machine architecture. Similar approaches in other fields are followed also in [14], [15]. The main subsystems of these models are hereinafter analysed.

The internal combustion engine (ICE) model, the source for the vehicle energy propulsion, uses the characteristic torque and BSFC maps at partial and full load, and its mechanical inertia. The model includes the control of the fuel flow including over run fuel cut off and idle speed control.

Hydraulic pumps and motors are modelled taking into account their inertia, and evaluating flow and mechanical losses through a map based approach. Their efficiency is computed as a function of the shaft speed and the difference of pressure between input and output. The ideal flow rate is determined by the shaft speed, pump displacement, and swash fraction, the latter only in case of variable displacement pumps, while the real flow rate comes from the ideal one plus the addition of leakage, function of inlet pressure and difference of pressure between input and output.

As regards check and relief valves, it suffices to describe the static (algebraic) input-output relationship based on the input and output port pressures, since the speed of response of the valves is many times faster than that of the overall system [9]. Additionally, the hydraulic spool valves, i.e. the components that control the actuators' operation, have been accurately modelled: in this regard a submodel of a 3 position 6 port hydraulic centre DC proportional valve has been defined and validated with experimental results. A simplified scheme of the valve is represented in Figure 3. 
The working machine kinematic model is defined considering the arm, bucket and joints dimension, mass and inertia, as resulted from the CAD models provided by the manufacturer.

The vehicle dynamics is studied considering its longitudinal behaviour. Although in the actual vehicle the motors drive independently the left and right driving wheels (i.e. during turning, these motors have different rotational speed due to different flow rate provided by the controlled pumps), in the models used for the study the two motors are considered to be rotating always simultaneously and steering was not considered.

The vehicle resistance is evaluated taking into account the usual term, composed by the rolling resistance and the aerodynamic drag (the latter can be neglected considering the extremely low vehicle speed), plus the addition of other contributions representative the effects of soil compaction, bulldozing and other ground interactions. The equation (1) explains all the above mentioned terms, where the $F_{\mathrm{ri}}$ 's are referred to the usual terms (respectively rolling, slope, aerodynamic), while the $F_{\text {si }}$ 's indicate the others (compaction, bulldozing, ground): as can be inferred from the proposed formulations, the additional terms are semiempirical. All the used parameters are shown in Table 2. Further details can be retrieved from [16].

$F_{\text {total }}=F_{\mathrm{rR}}+F_{\mathrm{r} \alpha}+F_{\mathrm{rA}}+F_{\mathrm{sC}}+F_{\mathrm{sB}}+F_{\mathrm{sG}}$

$F_{\mathrm{rR}}=f M g \cos \alpha$

$F_{\text {r } \alpha}=M g \operatorname{sen} \alpha$

$F_{\mathrm{rA}}=\frac{1}{2} \rho S C_{x} V^{2}$

$F_{\mathrm{sC}}=b\left(\frac{k_{\mathrm{c}}}{b}+k_{\varphi}\right)\left(\frac{z_{0}^{n+1}}{n+1}\right) C_{\mathrm{c}}$

$F_{\mathrm{sB}}=b\left(0.67 c z_{0} k_{\mathrm{pc}}^{\prime}+0.5 z_{0}^{2} \gamma_{\mathrm{s}} k_{\mathrm{p} \gamma}^{\prime}\right) C_{\mathrm{b}}$

$F_{\mathrm{sG}}=\frac{m g}{1000}\left(133+\frac{2.5}{3.6} v\right) C_{\mathrm{v}}$

Additionally, the vehicle mass taken as being variable during the vehicle operation, to take into account the effects of the bucket load.

\subsection{Results and validation}

Ihimer put at disposal of the study the standard version of AS12, equipped with sensors able to measure the pressure at inlet and outlet port of the hydraulic motors, the linear position of actuators of the arm and of the 
bucket, and the rotary velocity of left and right wheels. An acquisition board was installed on board vehicle, and two cameras shot the machine operation.

At first, the experimental activity made in collaboration with Ihimer had the main objective to define realistic duty cycles for propulsion and working operations [17]. The defined duty cycle shown in Figure 4, representing the skid-loader employed in a typical loading-unloading operation, can be divided into four main steps:

- Arrival at loading zone at maximum velocity.

- Actuation of the bucket and loading operation.

- Movement at maximum velocity towards unloading area.

- Actuation of arm and bucket for unloading.

Some of the results obtained from simulation are visible in Figure 5 and show the power flows of the internal combustion engine, the working hydraulic circuit, and the hydrostatic transmission. The plots in particular show the high fluctuation of the ICE power, that implies significant variation of its efficiency. The electric hybridization, as described later, can attenuate this problem, allowing the ICE to work constantly on or near its best efficiency zone.

Additionally, simulation results of the hydraulic circuit (i.e. input/output hydraulic motor pressure, input pressure on the distributor valve), illustrated in Figure 6, have been compared with the outputs from the experimental tests. In this regard, a comparison between experimental and simulated input/output pressure of an hydraulic motor, for the skid-loader performing a portion of the driving cycle of Figure 4 (i.e. composed by a sequence of acceleration, constant speed driving and braking), is shown in Figure 7 . The validation has been made in reference to the vehicle longitudinal behaviour, since the skid lateral dynamic is not simulated in the model. On the other hand, a comparison between experimental and simulated pressure at the input/output ports of the distributor valve, according to the nomenclature adopted in Figure 3, during a portion of the working cycle of Figure 4 (i.e. composed by the raising and subsequent lowering of the arm) is shown in Figure 8. Despite some noise and lag in acquisition values respect to the simulated ones, peak, mean values and waveforms proved to be consistent in the considered cases. 


\section{Hybrid propulsion system design}

The hybridization of the conventional system can be made following two main criteria: replacing the hydraulic components (i.e. the motors of hydrostatic transmission) with the corresponding electric ones, or electrifying a subset of components, leaving unmodified the others. The first approach was followed with reference to the propulsion system, substituting the whole hydraulic transmission (i.e. variable displacement pumps, valves, hydraulic motors) with electric drives. The other one was instead related to the working system, for which, in all the considered variants, some hydraulic components have been maintained unmodified (i.e. the hydraulic actuators of the working hydraulic circuit).

The following paragraphs show the preliminary design of the new hybrid powertrain architectures, the energy management strategy and the sizing of components from simulation results.

\subsection{Powertrain architectures}

As mentioned, one of the main goals of the activity has been to replace the hydrostatic transmissions with the adoption of much more efficient solutions, i.e. electric drives. At this way, the selected powertrain architectures are always of the series-hybrid type, since parallel hybrid schemes normally require to keep large part of the standard hydraulic or mechanical components. The needed power is firstly converted into electricity, and the sum of energy between the two power sources is made in terms of electric quantities in an electric node, commonly a DC bus. The presence of a Rechargeable Energy Storage System (RESS) allows regenerative braking and helps in reducing the size of the primary converter. Starting from this reference configuration, three versions have been developed. The following representations are simplified, since several details of the working hydraulic circuits of Figure 2 are not shown.

- Standard series hybrid, that requires separate electric drives for propulsion and working operations, as shown in Figure 9. In this configuration, the hydrostatic transmissions (i.e. variable displacement pumps and hydraulic motors) are replaced by two electric drives. On the contrary, the working hydraulic circuit was left unmodified (Figure 2), apart from the electrification of the hydraulic fixed gear pump. 
- Simplified series hybrid, in which the pump that feeds the hydraulic actuators is left mechanically linked to the ICE, as for the conventional hydraulic skid loader, while the propulsion electric drives are the same of the previous series hybrid configuration. The scheme is shown in Figure 10.

- Advanced series hybrid, equivalent to the standard series hybrid version with the presence of a redesigned working hydraulic circuit (Figure 11). In this regard it must be said that the standard working hydraulic circuit (Figure 2) is not able to recover the energy when lowering the arm, because of the presence of the distributor valve and of the irreversible pump. These components have been replaced by a new closed circuit provided with a reversible variable displacement pump, designed in cooperation with the manufacturer. This pump is in particular characterised by the capability to work also in condition of pressure and flow inversion; this fact, when the arm and the bucket are descending, allows to send back the energy flow towards the working electric drive, that is therefore able to work as generator recharging the on-board electrochemical storage system. However, several other hydraulic solutions can be considered, some of them reported in literature [1], [5], [20].

\subsection{Energy management strategy}

The Power Management Module determines, as a function of driver's requests and possible other signals (slope measure, ...), which part of the requested propulsive power must be delivered by the PC and which by the RESS. According to Figure 9 and equation (2), to determine how to decompose the quantity $P_{\mathrm{U}}(t)$ (useful power) into $P_{\mathrm{PC}}(t)$ and $P_{\mathrm{RESS}}(t)$.

$P_{\mathrm{U}}(t)=P_{\mathrm{ED}_{\mathrm{A}}}(t)+P_{\mathrm{ED}_{\mathrm{P}}}(t)+P_{\mathrm{AUX}}(t)=P_{\mathrm{PC}}(t)+P_{\mathrm{RESS}}(t)$

The purpose of determining the share of the useful power between the two sources is quite complex and has been described with more details in [15], [21], [18]. Here is reminded that:

- $\quad P_{\mathrm{EDp}}(t)$ is determined to answer the driver's commands as closely as possible. It is a direct consequence of the vehicle duty cycle and resistance load.

- $\quad P_{\mathrm{EDa}}(t)$ is determined to follow the working load duty cycle, reported as displacement of the linear actuators in function of the time.

- $\quad P_{\mathrm{AUX}}(t)$ is given by the auxiliary loads. 
- $\quad P_{\mathrm{PC}}(t)$ is determined by PMM according to some optimization rule.

- $\quad P_{\text {RESS }}(t)$ is automatically determined by difference.

It must also be noticed that the useful power that goes into the loads $P_{\mathrm{EDp}}(t), P_{\mathrm{EDa}}(t)$ and $P_{\mathrm{AUX}}(t)$ can be imagined to be constituted by an average value and ripple. Therefore it is possible to control the system such as the ripple $r(t)$ is delivered by the RESS and does not form part of the primary converter (3).

$$
\begin{aligned}
& P_{\mathrm{RESS}}(t)=P_{\mathrm{RESSa}}(t)+r(t) \\
& P_{\mathrm{PC}}(t)=P_{\mathrm{Ua}}(t)-P_{\mathrm{RESSa}}(t)
\end{aligned}
$$

Hence, the primary converter delivers only average powers: it works at fixed or slowly variable points. The RESS manages the ripple component $r(t)$ of the needed useful power, with the addition of the average power $P_{\text {RESSa }}(t)$ to maintain the SOC balanced during normal operating condition. The strategy presented above requires also the consideration of the future system load: the approximate level of power needed by the vehicle in future can be assumed to be equal to the mobile average of $P_{\mathrm{U}}(t)$ in the recent past, assuming that:

$$
P_{\mathrm{Ua}}(t)=\frac{1}{T} \int_{t-T}^{t} P_{\mathrm{U}}(\tau) d \tau
$$

After determining $P_{\mathrm{Ua}}(t)$ an internal algorithm is used to choose the optimal values of the ICE rotary velocity, corresponding to the minimum fuel consumption. The control arrangement is reported in Figure 12. Further details of the energy management strategy, also applied in a similar way to the other considered variants, are described also in [17].

Regarding the working electric drive, where present (i.e. Standard Series Hybrid and Advanced Series Hybrid), the two following control strategies have been adopted:

- Basic control, in which the electrified working pump is maintained always at its reference constant speed value.

- ON-OFF control, in which the electrified working pump is switched off during the transfers of the skidloader at its maximum speed, and consequently the movement of the arms is stopped. 


\subsection{Modelling subsystems}

Modelling activity has been performed in AMESim and Dymola environments, for all the three series hybrid versions. The vehicle and kinematic models have been left equal to those used for the conventional hydraulic working machine, while the working hydraulic circuit was modified according to the changes previously explained. Additionally, the electric components were defined according to the general criteria summarized below.

- As regards electric machines EM and EG, the used models replace the machines, their converter and the related control by physical models. A physical model of an asynchronous induction machine with integrated inverter and field oriented control, including voltage and current limitation as well as flux weakening, is used. The efficiency map is shown in Figure 13. As visible, the efficiency is equal to $92 \%$ at value of rated power. Having EM and EG machines different power levels, the efficiency map has been further downscaled, in reference to the machine nominal torque.

- The lithium battery is modelled through an equivalent electrical circuit, characterised by the presence of voltage source and an internal resistance. Its behaviour is defined taking as reference an LFP cell whose characteristics are in Table 3. The parameters and their dependency from SOC can be easily determined by some basic experimental tests, of the type described in [19]

\section{Results}

\subsection{Sizing}

Simulation results are related to the sizing of the three different hybrid configurations: the powertrains have been dimensioned in agreement with the duty cycle already described in Section 2. Starting from that, electric drives have been sized according the following criteria.

The propulsion electric drives, were sized to satisfy the vehicle maximum acceleration and speed in off-road driving condition. The working electric drive, where present, is instead sized considering required the maximum actuator force and power. 
The ICE is dimensioned to be able to fulfil the tasks coming from the realisation of the energy management strategy defined in Section 3, with the maximum average efficiency. The RESS is defined taking into account the minimum allowable voltage for the inverter, controlled through SV-PWM technique [22], and the current limits for the LFP cell used. The final sizing of the Standard series hybrid configuration is in Table 4.

Figure 14 shows the power flows (the meaning of the quantities is easily understood looking at Figure 9) on the defined duty cycle, always for the Standard series hybrid version. It clearly shows the share of the power between the two sources, according to equation (2): at the beginning of the simulation SOC decreases very rapidly, because of the small contribution by the ICE. Later, the situation stabilises and only small variation of SOC around its average value remains.

The limits of the battery current during charge and discharge provided by the manufacturer have been respected: the considered cell is in fact able to deliver a continuous current equal to $2 C_{\mathrm{n}}$ (where $C_{\mathrm{n}}$ is the nominal capacitance) during discharge, and up to $C_{\mathrm{n}}$ during charge. Furthermore,

Figure $\mathbf{1 4}$ shows that the battery SOC through the selected algorithms can be kept near the imposed reference value, with oscillations during charge-discharge, at regime, lower than $1 \%$.

The proposed usage of lithium battery could rise concerns about life: it must however be said, considering the available manufacturer's indications [23] and specific experiences made by the authors [24], that a battery subject to micro-cycles in which the SOC oscillates by $1-2 \%$ can reach one million. That, considering that we have, around ten micro-cycles/hour during the machine working hours, allows to hypothesize that the battery can reach the end of its calendar life, estimated in ten years, before its end of life due to microcycling.

The composition of the useful powers is instead visible in Figure 15: as had already been explained in Section $3, P_{\mathrm{EDp}}(t)$ is a direct consequence of the velocity profile, while $P_{\mathrm{EDa}}(t)$ is determined by the need to be able to follow the working load duty cycle.

In the Standard series hybrid configuration the picture in Figure 15 is referred to, the power required by the hydraulic pump that feeds the working hydraulic circuit is always positive, therefore it is not possible to 
recover any energy during the arm lowering. This fact is caused by the presence of not reversible hydraulic components; it can be however overcome by adopting the Advanced series hybrid configuration as already mentioned. A totally different situation can be retrieved from the analysis of the power fluxes of the propulsion electric drive, that during breaking is negative and can be recovered inside the RESS.

\subsection{Fuel consumption}

The simulation results are able to estimate also the fuel consumption outputs for the three cases analysed. The fuel consumption map for the original ICE has been obtained downscaling the characteristic curves at full load. Starting from the existing map, because of the difficulty to have access to specific fuel consumption maps for smaller engines, the maps for the hybrid versions were further downscaled. The engine torque was scaled down, by leaving the speed range unchanged. The so "scaled down" engine was assumed to have the same specific fuel consumption values as the bigger one. The obtained results are reported in Table 5, and show the fuel saving for the three different levels of hybridization. The Simplified series hybrid version is able to ensure a fuel consumption reduction by about $11 \%$, while the Standard series hybrid is $14 \%$. The Advanced series hybrid configuration, which because of the presence of a redefined hydraulic circuit is able to recover the energy during the arm lowering, can guarantee a fuel reduction by about $25 \%$.

As already noted, the flexibility allowed by the presence of the working electric drive might also guarantee much more flexibility in the management of the fixed gear hydraulic pump, allowing further fuel saving. In this regard, in addition to the Basic control in which the pump is maintained at its nominal velocity, also the ON/OFF control has been evaluated. This logic, as previously explained, allows to switch off the working electric drive and stop the hydraulic pump, when the skid loader is moving at its maximum velocity. It is indeed possible to avoid the baseline constant absorption of power, of around $2.5 \mathrm{~kW}$, depicted in Figure 15 (ED arm/bucket power curve).

A fuel consumption evaluation due the usage of this technique is presented in Table 5 as well: the start and stop transients of the electrified pump have been not considered, the ICE load variation as defined in (4) was 
taken into account; the fuel consumption $f_{c}$ due the application of the ON/OFF logic was calculated according to the following expression:

$$
f_{c}=f_{\mathrm{cAO}} \frac{T_{\mathrm{wo}}}{T_{t}}+f_{\mathrm{cOFF}} \frac{T_{\mathrm{ms}}}{T_{t}}
$$

Where:

- $f_{\mathrm{cAO}}$ is the simulated, hourly fuel consumption when electrified working pump is always ON, and working at its nominal speed.

- $f_{\text {cOFF }}$ is the simulated, hourly fuel consumption when electrified working pump is always OFF.

- $\quad T_{\mathrm{ms}}$ is the time corresponding to the transfers at the skid loader maximum speed.

- $T_{\text {wo }}$ is the time corresponding to the rest part of the imposed duty cycle (i.e. working operations, $\ldots$ ).

- $T_{\mathrm{t}}$ represents the total duration of the imposed duty cycle.

The results show that a shutdown of the working electric drive is able to produce a further reduction of consumption by around $10 \%$ for the Standard series hybrid configuration, and $7 \%$ for the Advanced series hybrid one.

\subsection{Costs evaluation}

Although the vehicle is at prototype level, an industrial cost analysis divided in capital and running costs for the different configurations analysed has been carried out. The following criteria have been used:

- An ICE cost of $150 € / \mathrm{kW}$ was assumed, for both the engine used in the conventional and hybrid skid loaders.

- An hydraulic transmission cost of $350 € / \mathrm{kW}$ was assumed, both for propulsion and working hydraulic systems. An additional development cost of $10 \mathrm{k} €$ has been considered for the design of the new working hydraulic circuit proposed in the Advanced series hybrid configuration.

- A RESS cost of $400 € / \mathrm{kWh}$ was assumed, including the Battery Management System (BMS). 
- As far as the electric drives ED and EG are concerned, their components price, commercially available, have been evaluated in $200 € / \mathrm{kW}$ for the electric machine and $50 € / \mathrm{kW}$ for the converter.

- In terms of running cost, a standard scenario in which the skid loader stays in service for about 1000 hours per year has been assumed. The fuel consumptions was obtained from simulation results (Table 5), and the fuel cost was taken equal to $1.8 € / \mathrm{L}$.

- The maintenance costs have been defined in two different ways: equal to $1300 € / \mathrm{y}$ for the conventional version; equal to $600 € / y$, due the reduction of the hydraulic components, for all the hybrid variants.

- Substitution of components for aging has been not considered, both for the conventional skid-loader and for the hybrid variants. In this regard, a specific evaluation about the RESS is reported in paragraph 4.1.

Cost analysis for all the examined configurations is detailed in Table 6. Starting from that, NPV, IRR and Payback Time have been evaluated, considering an interest rate of $4 \%$. The results of the analysis are shown in Table 7 and can be summarized as follows:

- All the examined hybrid variants show a payback time much lower than the whole life of the skid loader, estimated in ten years.

- The Simplified series hybrid configuration shows the best cost competitiveness, because of the reduced number of electrified components (the working electric drive is absent), in conjunction with the possibility to adopt, as for the more sophisticated versions, an effective energy management strategy.

- The Standard series hybrid, because of the additional cost related to the working electric drive, is less competitive than the previous one, despite of the higher fuel consumption saving. On the other hand, this variant becomes competitive when additional control strategies for the pump are introduced. In particular it was shown that switching off the pump when the vehicle is at its maximum speed allows, under the considered duty cycle, to make the payback time almost equivalent to the Simplified series hybrid configuration one.

- The Advanced series hybrid variant, that uses the same electrified components of the Standard series hybrid skid loader, presents the main drawback of the hardware and development cost of the new hydraulic circuit, that is needed to send back the energy during the lowering of the arms. The additional 
fuel consumption reduction, also taking into account the ON/OFF control strategies for the pump, is therefore not able to compensate the extra costs.

\section{Conclusions}

In conclusion, the process of modelling and sizing of several variants of an hybrid skid loader has been discussed in detail and performed in a systematic approach, starting from modelling and experimental validation of the conventional, no hybridised version.

The results have shown the general feasibility of hybridisation, producing fuel saving from $11 \%$ up to $31 \%$, depending on the configuration and on the control strategy adopted. In fact, all the examined configurations are cost-effective, since they have payback times much lower than the useful life of the working machine.

In particular, the results have shown a distinct advantage of the simplified hybrid configuration, characterised by the highest number of hydraulic components that remain unmodified respect to the conventional skid loader, or for the fully electrified version, when a suitable control strategy is applied to the electrified pump.

Further developments should be aimed to the realisation of an hybrid machine prototype, by Ihimer. This should allow also to validate the hybrid simulation model by experimental results, analysing also the effects of the real driver behaviour on energy consumptions. 


\section{Bibliography}

[1] T. Lin, Q. Wang, B. Hu, W. Gong, Research on the energy regeneration systems for hybrid hydraulic excavators, J. Automation in Construction, vol. 19 (2010), pp. 1016-1026.

[2] S. Hui, J. Junqing, Research on the system configuration and energy control strategy for parallel hydraulic hybrid loader, J. Automation in Construction, vol. 19 (2010), pp. 213-220.

[3] Q. F. Wang, Y. T. Zhang, Q. Xiao, Evaluation for energy saving effect and simulation research on energy saving of hydraulic system in hybrid construction machine, Chinese Journal of Mechanical Engineering, vol. 41 (2005), pp. 135-140.

[4] T. O. Andersen, M. R. Hansen, H. C. Pedersen, F. Conrad, Regeneration of Potential Energy in Hydraulic Forklift Trucks, in: $6^{\text {th }}$ International Conference on Fluid Power Transmission and Control, Hangzhou, 2005, pp. 302-306.

[5] T. Minav, L. Laurila, J. Pyrhonen, Energy Recovery Efficiency Comparison in an Electro-hydraulic Forklift and in a Diesel Hybrid Heavy Forwarder, International Symposium on Power Electronics, Electrical Drives, Automation and Motion (SPEEDAM 2010), Pisa, 2010.

[6] AMESim official site (http://www.plm.automation.siemens.com/en_us/products/lms/imaginelab/amesim/index.shtml), date of access: 15-06-2015.

[7] Dymola official site (http://www.3ds.com/products-services/catia/products/dymola), date of access: 15-06-2015.

[8] Modelica Association official site (https://modelica.org), date of access: 15-06-2015.

[9] Modelon AB, Hydraulics Library Manual and Tutorial, 2011.

[10] A. Kugi, K. Schlacher, H. Aitzetmuller, G. Hirmann, Modelling and simulation of a hydrostatic transmission with variable-displacement pump, Mathematics and Computers in Simulation, vol. 53 (2000), pp. 409-414. 
[11] N. Manring, G. Luecke, Modeling and designing a hydrostatic transmission with a fixed-displacement motor, Journal of Dynamic Systems, Measurement, and Control, vol. 120 (1998), pp. 45-49.

[12] M. Comellas, J. Pijuan, X. Potau, M. Nogués, J. Roca, Analysis of a hydrostatic transmission driveline for its use in off-road multiple axle vehicles, Journal of Terramechanics, vol. 49 (2012), pp. 245- 254.

[13] R. Paoluzzi, G. Zarotti, The minimum size of hydrostatic transmission for locomotion, Journal of Terramechanics, vol. 50 (2013), pp. 153-164.

[14] D. Poli, A. di Donato, G. Lutzemberger, Experiences in Modeling and simulation of hydrogen fuel-cell based propulsion systems, SAE Technical Paper (2009), 2009-24-0084, issn: 0148-7191, doi: 10.4271/200924-0084.

[15] G. Lutzemberger, G. Pede, G. Sanna, H. Tarun, Systematic development of series-hybrid bus through modelling, Vehicle Power and Propulsion Conference (VPPC 2010), Lille (France), 2010, isbn: 978-1-42448220-7, doi: 10.1109/VPPC.2010.5729243.

[16] M. Ehsani, Y. Gao, A. Emadi, Modern Electric, Hybrid Electric and Fuel Cell Vehicles, CRC Press, 2010.

[17] A. Bertini, M. Ceraolo, G. Lutzemberger, Development of a hybrid skid loader through modelling, Energy Conference and Exhibition (ENERGYCON), 2012 IEEE International, 9-12 Sept. 2012, Florence, isbn: 978-1-4673-1453-4, doi: 10.1109/EnergyCon.2012.6347719.

[18] M. Ceraolo, A. di Donato, C. Miulli, G. Pede, Microcycle-based efficiency of hybrid vehicle batteries, IEEE Vehicular Power and Propulsion Conference (VPPC 2005), Chicago (USA), 2005, doi: 10.1109/VPPC.2005.1554533.

[19] M. Ceraolo, G. Lutzemberger, T. Huria, Experimentally-Determined Models for High-Power Lithium Batteries, SAE Technical Paper (2011), 2011-01-1365, issn: 0148-7191, doi: 10.4271/2011-01-1365.

[20] T. Minav, A. Virtanen, L. Laurila, J. Pyrhonen, Storage of energy recovered from an industrial forklift, J. Automation in Construction, vol. 22 (2012), pp. 506-515. 
[21] M. Ceraolo, A. di Donato, G. Franceschi, A General Approach to Energy Optimization of Hybrid Electric Vehicles, IEEE Transaction on Vehicular Technology vol. 57 n. 3 (2008), 1433-1441.

[22] N. Mohan, Power electronics, Wiley, 2012.

[23] M. Ceraolo, G. Lutzemberger, M. Marracci, High power lithium batteries usage in hybrid vehicles, Vehicle Power and Propulsion Conference (VPPC), 2010, IEEE Lille (France) 1-3 Sept. 2010, isbn 978-14244-8220-7, doi: 10.1109/VPPC.2010.5729096.

[24] A123 official site (http://www.a123systems.com/solutions-transportation.htm), date of access 15-062015. 
Table list

Table 1: General characteristics of the hydraulic skid-loader

Table 2: Resistance force parameters

Table 3: LFP cell characteristics

Table 4: Sizing of the standard series hybrid configuration

Table 5: Fuel consumption results for the three hybrid variants; standard and advanced variants with ON-OFF control of the working electric drive

Table 6: Components and usage cost analysis

Table 7: NPV, IRR, Payback time evaluation 
Figure captions

Figure 1: Ihimer AS12 skid loader

Figure 2: Conventional powertrain scheme

Figure 3: Simplified scheme of the distributor valve

Figure 4: Cycles for propulsion and working operations

Figure 5: Power flows, conventional skid loader

Figure 6: Pressure at input/output of hydraulic motors (top), and on spool distributor valves (bottom)

Figure 7: Detail of simulated and measured pressure at input/output of the hydraulic motor

Figure 8: Detail of pressure at input (port $P$ ) and output (port $A$ ) of the distributor valve

Figure 9: Standard series hybrid configuration

Figure 10: Simplified series hybrid configuration

Figure 11: Working hydraulic circuit of the advanced series hybrid version

Figure 12: Energy management strategy implemented in the PMM

Figure 13: EM efficiency map

Figure 14: Main power flows, standard series hybrid skid loader

Figure 15: Useful power, standard series hybrid skid loader 